fatores que influenciam nos resultados não permitem que se chegue a esta conclusão. A IG neste estudo foi determinada tardiamente pela ultra-sonografia. Já no estudo de Fescina et al. ${ }^{5}$, o cálculo da IG foi apenas baseado na data da última menstruação (DUM). O mais apropriado seria basear-se na DUM correspondente à ultra-sonografia realizada até a $12^{a}$ semana. Na ausência da DUM, o correto seria considerarem-se pelo menos duas ultra-sonografias compativeis até a $20^{\mathrm{a}}$ semana. Sem estes cuidados é impossivel datar corretamente a gestação e o diagnóstico de adequação antenatal e pós-natal fica prejudicado. A avaliação neonatal da idade gestacional que é feita em geral com $24 \mathrm{~h}$ de vida (método de Capurro, por exemplo) apresenta maior erro quando comparada à ultra-sonografia em IG precoce. O diagnóstico definitivo de AIG, PIG ou GIG é dado pela curva de crescimento adotada pelo berçário. No estudo em análise não se fez referência à curva adotada. Para interpretar os valores obtidos de AU, além dos aspectos considerados, deve-se considerar a técnica de medida utilizada. No estudo não se destacou qual foi a técnica utilizada - a que é realizada da sínfise púbica ao fundo uterino, fixando-se a fita métrica com a face cubital da mão, aquela em que a leitura é realizada com a fita entre os dedos indicador e médio ou a técnica do arco, com a fita fixada entre os dedos polegar e indicador? Foi considerado um número mínimo de medidas (consultas)? As gestantes esvaziavam a bexiga antes do procedimento de medida da AU? Como era a fita métrica - flexivel ou inelástica? Além disso, o fato de a medida da AU ter sido realizada por vários observadores é um dos fatores que pode ter determinado grande variação nas medidas. Tomou-se alguma medida para diminuir a variabilidade intra-observador?

Como vários outros estudos já demonstraram, em virtude dos inúmeros fatores epidemiológicos que influenciam o crescimento fetal, cada região deve ter a sua própria curva de AU. No entanto, na construção destas curvas os fatores acima descritos que podem levar a erros de interpretação devem ser rigorosamente controlados.

\title{
Referências
}

1. Rumbolz WL, McGoogan LS. Placental insufficiency and the small undernourished full-term infant. Obstet Gynecol. 1953;1(3):294-301.

2. American College of Obstetricians and Gynecologists. Intrauterine growth restriction. Washington D.C: ACOG; 2000. (ACOG Practice Bulletin, 12).

3. Royal College of Obstetricians and Gynaecologists. The Investigation and management of the small-for-gestationalage fetus. London: RCOG; 2002. (Guideline, 31).

4. Oppermann MLR, Duncan BB, Mengue SS, Ramos JGL, Serruya SJ, Schmidt MI. Distribuição da altura uterina ao longo da gestação em uma corte brasileira - comparação com a curva de referência do Centro Latino-Americano de Perinatologia (CLAP). Rev Bras Ginecol Obstet. 2006;28(9):513-22.

5. Fescina RH, Quevedo C, Martell M, Nieto F, Schwarcz R. Altura uterina como método para predecir el crescimiento fetal. Bol Oficina Sanit Panam. 1984;96(5):377-86.

6. Freire DMC, Paiva CSM, Coelho EAC, Cecatti JG. Curva da altura uterina por idade gestacional em gestantes de baixo risco. Rev Bras Ginecol Obstet. 2006;28(1):3-9.

\section{Comentários do autor}

O Conselho Editorial da RBGO acertou ao reconhecer a importância do tema abordado em nosso artigo $^{1}$, redigindo um comentário editorial a respeito no mesmo fascículo². Ao fazê-lo, toma como referência o artigo de Chauhan e Magann ${ }^{3}$, publicado recentemente, que compara as propriedades diagnósticas da altura uterina na detecção da restrição do crescimento intra-uterino obtidas no estudo do American College of Obstetricians and Gynecologists (ACOG) e no do Royal College of Obstetricians and Gynaecologists (RCOG). Os resultados muito díspares desses dois estudos ilustram a problemática da medida rotineira da altura uterina usada no cuidado pré-natal: considerando uma probabilidade pré-teste de $10 \%$ para restrição do crescimento intra-uterino, as probabilidades pós-teste positivo (equivalentes aos valores preditivos positivos) foram de $66-70 \%$ (ACOG) e 20\% (RCOG).

O comentário editorial analisa criticamente nosso artigo e o estudo de Fescina et al. ${ }^{4}$, que 
derivou a curva atualmente recomendada pelo Ministério da Saúde ${ }^{5}$. Agradecemos a oportunidade de manifestar nossa visão sobre as questões abordadas e suas implicações.

Primeiramente, é preciso ressaltar que o Estudo Brasileiro de Diabetes Gestacional ${ }^{6}$ (EBDG), base populacional empregada em nosso artigo ${ }^{1}$, é um estudo de coorte multicêntrico que acompanhou o cuidado obstétrico de mais de 5000 gestantes atendidas em serviços de 4 regiões brasileiras entre 1991 e 1995. Por ser um estudo observacional, o protocolo não visou intervir nas rotinas pré-natais desses serviços (com exceção da padronização do teste de tolerância à glicose, irrelevante para a análise atual). Por essa razão, como descrito na seção de Métodos, o estudo visou avaliar a medida de altura uterina, como efetuada nas rotinas prénatais vigentes nesses serviços. Assim, embora os métodos de medida da altura uterina possam ter variado entre os centros estudados, em consonância com o objetivo do estudo, optou-se por validar esse uso, como efetuado em serviços do SUS. Ou seja, aceitando as medidas de altura uterina exatamente como realizadas nos ambulatórios do nosso país, com as variações pertinentes ao método.

As diretrizes para o SUS $^{5}$ baseiam-se em curva de crescimento de altura uterina derivada do estudo de Fescina et al. ${ }^{4}$, um estudo com amostra pequena e selecionada de gestantes uruguaias, mas com medidas altamente padronizadas de altura uterina. Estudos como esse de Fescina et $\mathrm{al} .{ }^{4}$ poderiam ser vistos como estudos de eficácia diagnóstica, isto é, capazes de mostrar que a altura uterina, medida de forma muito padronizada, é capaz de detectar desfechos adversos da gravidez. Cabe ressaltar, contudo, que o tamanho pequeno da amostra do estudo de Fescina et al. ${ }^{4}$ limitou sua capacidade de avaliar a eficácia preditiva da altura uterina, expressa, por exemplo, por uma sensibilidade de $52 \%$, desacompanhada de intervalos de confiança, seguramente amplos.

Por analogia, o nosso estudo expressa a efetividade, isto é, a avaliação da capacidade diagnóstica da medida da altura uterina como correntemente empregada no SUS. Nesse sentido, foi além de dois outros estudos publicados no Brasil recentemente ${ }^{7,8}$. Além disso, é hoje bem reconhecido que um método diagnóstico proposto a partir de dados de uma determinada amostra precisa ser validado em outras amostras ${ }^{9}$. Ao testar a curva derivada por Fescina et $a{ }^{4}{ }^{4}$ na população de gestantes brasileiras a sensibilidade do percentil 10 de altura uterina alcançou, no máximo, 6\% (IC95\% = 0,3-11) na detecção de recém-nascido pequeno para idade gestacional, o que é bem diferente dos $52 \%$ obtidos quando a sensibilidade foi avaliada na amostra de origem.
No editorial se questiona como foram definidos os recém-nascidos pequeno e grande para idade gestacional em nosso estudo. Como relatado no artigo, as definições foram obtidas dos percentis $10 \mathrm{e}$ 90, respectivamente, das distribuições dos pesos ao nascer específicos para sexo do recém-nascido e para a semana gestacional de nascimento. $O$ tamanho e abrangência da amostra do EBDG permitiram a construção desses pontos de corte.

Concordamos com o comentário editorial, quando aponta as limitações das estimativas de idade gestacional obtidas nos dois artigos. Por exemplo, a data da última menstruação (DUM), isoladamente, foi empregada na construção das duas curvas de peso neonatal freqüentemente utilizadas nos centros de neonatologia, as curvas de peso neonatal da população americana publicadas por Lubchenco et al. ${ }^{10}$ e Alexander et al. ${ }^{11}$. No artigo de Fescina et al. ${ }^{4}$, a idade gestacional foi determinada apenas pela DUM. No nosso artigo, ela foi estimada pela ultra-sonografia, a partir do seguinte construto: em $78,6 \%$ da população estudada a ultra-sonografia foi realizada antes da $26^{\mathrm{a}}$ semana e essa idade gestacional foi tomada como referência para estimar idade gestacional ao longo da gravidez; em 21,4\%, a ultra-sonografia foi realizada após a $26^{\mathrm{a}}$ semana e, nesses casos, a idade gestacional assim definida só foi considerada nas análises quando consistente com DUM ou com o exame neonatal de Capurro.

Essas limitações foram reconhecidas na Discussão de nosso artigo, mas é importante não perder o foco sobre o alcance dos objetivos e as implicações práticas dos resultados obtidos. Nesse sentido, deve ser frisado que as curvas hoje usadas nos serviços de saúde, construídas a partir de amostra pequena de gestantes, estudadas em centro de referência, décadas atrás em outro país, não refletem a realidade atual do cuidado pré-natal básico em nosso país. A Figura 2 de nosso artigo ilustra bem o fato de que as alturas uterinas obtidas no estudo de Fescina et al. ${ }^{4}$ são sempre menores do que as das brasileiras, a ponto de, da $30^{\mathrm{a}}$ semana em diante, a curva do p90 daquele estudo aproximar-se mais à curva p10 do que à curva p90 das brasileiras. Todas as diferenças nas propriedades diagnósticas (sensibilidade, especificidade e valores preditivos) relatadas são decorrentes desse fato. Como visto nas Tabelas 2 e 3 de nosso artigo, as curvas de Fescina et al. ${ }^{4}$ identificaram apenas $1 \%$ das gestantes brasileiras como em risco para baixo peso ao nascimento e por volta de $50 \%$ como em risco para grande peso ao nascimento; os percentís 10 e 90 daquele estudo, se bem calibrados para aplicação na população brasileira, deveriam identificar aproximadamente $10 \%$ das gestantes em ambos os casos. Essas diferenças, bem mais do que os possiveis erros de classificação (idade gestacional, 
altura uterina ou pequeno/grande peso ao nascer) no EBDG, são as responsáveis pelo desempenho insatisfatório da curva de Fescina et al. ${ }^{4}$ quando aplicada em gestantes brasileiras: sensibilidade do p10 para a detecção de pequeno para a idade gestacional de 1-6\% e especificidade do p90 para a identificação de grande para a idade gestacional de 45-60\%, dependendo da semana gestacional.

Nosso estudo não foi delineado primariamente para estabelecer, com precisão, uma nova curva de altura uterina brasileira. Ao usar dados secundários, no entanto, conseguiu, pela abrangência e tamanho da amostra, mostrar as limitações da curva atualmente empregada no SUS. Além disso, a curva do nosso estudo, por ter sido derivada de gestantes brasileiras de várias regiões, fornece um quadro real da validade das medidas de altura uterina corren- temente empregadas no SUS. Dessas curvas, alguns achados merecem ser ressaltados. O p10 do nosso estudo mostrou uma sensibilidade máxima de 29\% (IC95\% = 18-40) na detecção de recém-nascido pequeno para idade gestacional, o que é inadequado para um teste de rastreamento. O uso do percentil 25 melhorou a sensibilidade para 49\% (IC95\% $=37-60)$, mas ainda assim, ela ficou aquém daquela esperada para um bom teste de rastreamento. Nossos dados e os das publicações prévias em populações brasileiras demonstram a necessidade de nova curva de crescimento da altura uterina, capaz de efetivamente subsidiar decisões preventivas no cuidado pré-natal.

Maria Lúcia Rocha Oppermann

\section{Referências}

1. Opperman ML, Duncan BB, Mengue SS, Ramos JGL, Serruya SJ, Schmidt MI. Distribuição da altura uterina ao longo da gestação em uma coorte brasileira - comparação com a curva de referência do Centro Latino-Americano de Perinatologia (CLAP). Rev Bras Ginecol Obstet. 2006; 28(9): 513-22.

2. Bittar RE. Distribuição da altura uterina ao longo da gestação em uma coorte brasileira - comparação com a curva de referência do Centro Latino-Americano de Perinatologia [editorial]. Rev Bras Ginecol Obstet. 2006; 28(9): 509-10.

3. Chauhan SP, Magann EF. Screening for fetal growth restriction. Clin Obstet Gynecol. 2006;49(2): $284-94$.

4. Fescina RH, Quevedo C, Martell M, Nieto F, Schwarcz R. Altura uterina como método para predecir el crescimiento fetal. Bol Oficina Sanit Panam. 1984;96(5):377-86.

5. Ministério da Saúde. Assistência pré-natal: manual técnico. 3a ed. Brasília: Secretaria de Políticas de Saúde; 2000. p. 39-41.

6. Schmidt MI, Duncan BB, Reichelt AJ, Branchtein L, Matos MC, Costa e Forti A, et al. Gestational diabetes mellitus diagnosed with a 2-h 75-g oral glucose tolerance test and adverse pregnancy outcomes. Diabetes Care. 2001;24(7):1151-5.

7. Freire DMC, Paiva CSM, Coelho EAC, Cecatti JG. Curva da altura uterina por idade gestacional em gestantes de baixo risco. Rev Bras Ginecol Obstet. 2006;28(1):3-9.

8. Martinelli S, Bittar RE, Zugaib M. Proposta de nova curva de altura uterina para gestações entre a $20^{a}$ e $42^{a}$ semana. Rev Bras Ginecol Obstet. 2001;23(4):235-41.

9. Bossuyt PM, Reitsma JB, Bruns DE, Gatsonis CA, Glasziou PP, Irwig LM, et al. Towards complete and accurate reporting of studies of diagnostic accuracy: the STARD initiative. Fam Pract. 2004;21(1):4-10.

10.Lubchenco LO, Hansman C, Dressler M, Boyd E. Intrauterine growth as estimated from liveborn birth-weight data at 24 to 42 weeks of gestation. Pediatrics. 1963;32:793-800.

11.Alexander GR, Himes JH, Kaufman RB, Mor J, Kogan M. A United States national reference for fetal growth. Obstet Gynecol. 1996;87(2):163-8. 